Caroline Di Bernardi Luft

Sabrina de Oliveira Sanches

Giovana Zarpellon Mazo

Alexandro Andrade

Mestrado em Ciências do Movimento Humano. Centro de Educação Física, Fisioterapia e Desportos. Universidade do Estado de Santa Catarina. Florianópolis, SC, Brasil

Correspondência | Correspondence:

Caroline Di Bernardi Luft

R. Dr. Abel Capela, 135, ap.304 - Coqueiros 88080-250 Florianópolis, SC, Brasil

E-mail: caroluft21@hotmail.com

\section{Versão brasileira da Escala de Estresse Percebido: tradução e validação para idosos}

\author{
Brazilian version of the Perceived \\ Stress Scale: translation and \\ validation for the elderly
}

RESUMO

OBJETIVO: Traduzir a Escala de Estresse Percebido para a língua portuguesa do Brasil e verificar sua validade para mensurar o estresse percebido de idosos brasileiros.

MÉTODOS: A escala foi traduzida e testada em sua versão completa, com 14 questões e na reduzida, com dez questões. A tradução obedeceu às etapas de tradução, tradução reversa e revisão por um comitê. A escala traduzida foi aplicada, por meio de entrevista, a 76 idosos com idade média de 70,04 anos ( $\mathrm{DP}=6,34$; mín: 60; máx: 84 ). A consistência interna foi verificada por meio do coeficiente alfa de Cronbach e a validade de construto, por análise fatorial exploratória com rotação ortogonal pelo método varimax. As médias das versões completa e reduzida foram analisadas comparando o estresse percebido em função da auto-avaliação da saúde, nível econômico percebido, estado civil, condições de residência, entre outras.

RESULTADOS: Quanto à confiabilidade, a versão completa apresentou consistência interna semelhante $(\mathrm{r}=0,82)$ à reduzida $(\mathrm{r}=0,83)$. A análise fatorial revelou a existência de dois fatores para a completa e um para a reduzida. A questão 12 apresentou as menores cargas fatoriais. Ao analisar a possibilidade de a escala diferenciar o estresse percebido em função das variáveis, verificouse que a versão completa obteve maiores diferenças no estresse do que a reduzida.

CONCLUSÕES: A Escala de Estresse Percebido mostrou-se clara e confiável para mensurar o estresse percebido de idosos brasileiros, apresentando qualidades psicométricas adequadas.

DESCRITORES: Idoso. Estresse psicológico, diagnóstico.

Envelhecimento, psicologia. Testes psicológicos. Sensibilidade e especificidade. Validade dos testes. Tradução (Processo). Brasil. Saúde do idoso. 


\section{ABSTRACT}

OBJECTIVE: To translate the Perceived Stress Scale into Brazilian Portuguese, and to assess its validity for measuring perceived stress of Brazilian elderly.

METHODS: The scale was translated and tested in its full version including 14 questions and in a shortened version including ten questions. The whole translation process consisted of translation, back-translation and committee review. The translated version was applied, by means of interview, to 76 elders aged on average 70.04 years $(\mathrm{SD}=6.34$; range: $60-84)$. The internal consistency was verified by means of the Cronbach's alpha coefficient and the construct validity was analyzed by means of factorial exploratory analysis with varimax rotation. Full and shortened score means were analyzed comparing the perceived stress in terms of self-assessment of health, perceived socioeconomic condition, marital status, and living conditions, among others.

RESULTS: As for reliability, the full version has showed similar internal consistency $(\mathrm{r}=0.82)$ compared to the shortened one $(\mathrm{r}=0.83)$. The factorial analysis found two factors for the full and one factor for the shortened scale. Question 12 showed the lowest factorial loads. When analyzing PSS likelihood of differentiating the perceived stress in terms of the study variables, it was found the full scale had greater differences in perceived stress than the shortened scale.

CONCLUSIONS: The Perceived Stress Scale proved to be a clear and reliable tool to measure the perceived stress of Brazilian elderly, showing suitable psychometric performance.

KEY WORDS: Aged. Stress, Psychological, diagnosis. Aging, psychology. Psychological tests. Sensitivity and specificity. Validity of tests. Translating. Brazil. Health of the elderly.

\section{INTRODUÇÃO}

Os dados do último censo revelam que a população idosa no Brasil está aumentando. No entanto, a transição demográfica e epidemiológica brasileira não foi acompanhada de melhorias nas condições socioeconômicas e de assistência à saúde da população. $\mathrm{O}$ idoso brasileiro experimenta muitas dificuldades, desde questões como suporte social inadequado até o deficiente atendimento de saúde. $^{12}$

A burocracia da aposentadoria, as dificuldades financeiras, os problemas com o atendimento de saúde, morte de amigos e familiares e a discriminação social tornam o idoso cada vez mais susceptível ao estresse e à depressão. Talvez estes problemas estejam na origem da alta prevalência de depressão encontrada nesta população. ${ }^{10,12,20,21}$

Algumas pesquisas têm indicado que a ansiedade, depressão e estados de humor são variáveis que influenciam no desenvolvimento de doenças, como as cardiovasculares, ${ }^{5,18}$ ou mesmo as cognitivas. ${ }^{15,19}$ No entanto, o estresse, considerado precursor de diversas doenças, ${ }^{13}$ não tem recebido atenção nos estudos com idosos no Brasil.

Para os idosos, os agentes estressores podem ser os mais variados, como a aposentadoria, a morte de entes queridos, mudanças de papéis sociais, entre outros. ${ }^{6} \mathrm{No}$ entanto, a forma como o idoso percebe estes estressores é um dos principais determinantes de como ele é afetado pelo estresse. Desta forma, investigar apenas os fatores estressantes na vida do idoso pode não ser uma boa medida para saber o quanto ele é afetado por este. ${ }^{1,2}$

De acordo com Cohen \& Williamson² (1988), existem três formas de medir o estresse. A primeira é direcionada à presença de agentes estressores específicos; a segunda, aos sintomas físicos e psicológicos do estresse e a terceira, pretende mensurar a percepção de estresse individual de forma global, independente dos agentes estressores. Existem também escalas que mensuram o nível de estresse por meio de outras escalas que quantificam o impacto de eventos estressores específicos (life-events impact). Porém, esses 
instrumentos apresentam limitações, uma vez que os eventos relacionados ao estresse podem variar muito entre indivíduos.

Assim, Cohen et al' (1983) propuseram uma escala que mensura o estresse percebido, ou seja, mede o grau no qual os indivíduos percebem as situações como estressantes. Esta escala, denominada Perceived Stress Scale (PSS - Escala de Estresse Percebido), foi inicialmente apresentada com 14 itens (PSS 14), sendo também validada com dez (PSS 10) e quatro questões (PSS 4). A versão com quatro questões é utilizada em pesquisas por telefone. Os itens foram designados para verificar o quanto imprevisível, incontrolável e sobrecarregada os respondentes avaliam suas vidas. ${ }^{1}$ Estes três fatores tem sido considerados como componentes centrais na experiência de estresse. ${ }^{9}$ A PSS é uma escala geral, que pode ser usada em diversos grupos etários, desde adolescentes até idosos, pois não contém questões específicas do contexto. A ausência de questões específicas de contexto é um fator importante na escala e, provavelmente, a razão pela qual esta escala tenha sido validada em diversas culturas. ${ }^{1,2,8,11,14}$

O elevado nível de estresse percebido, mensurado por meio da PSS, está diretamente associado com desequilíbrios fisiológicos, como altos níveis de cortisol, triglicérides, interleucina-6 (IL-6), entre outros. ${ }^{4}$ Pesquisas como essa têm gerado aumento do interesse dos pesquisadores sobre o tema. No entanto, para a realização de tais estudos em uma perspectiva epidemiológica, é necessário um instrumento que demande pouco tempo para o seu preenchimento, mas que tenha características psicométricas satisfatórias. Além disto, esse instrumento deve avaliar o estresse dentro de uma perspectiva genuinamente internacional, característica importante na comparação de dados de pesquisa.

Considerando a necessidade de mensuração do estresse percebido de idosos e da falta de instrumentos em português que possam suprir esta necessidade, a presente pesquisa teve por objetivo realizar a tradução e validação da $\operatorname{PSS}^{1}$ (1983) para a língua portuguesa, verificando a clareza e a validade da tradução para idosos brasileiros.

\section{MÉTODOS}

O estudo foi realizado em duas etapas, a primeira com o objetivo de tradução e a segunda de aplicação da PSS traduzida.

A PSS possui 14 questões com opções de resposta que variam de zero a quatro $(0=$ nunca; $1=$ quase nunca; $2=$ às vezes; $3=$ quase sempre $4=$ sempre). As questões com conotação positiva $(4,5,6,7,9,10$ e 13$)$ têm sua pontuação somada invertida, da seguinte maneira, $0=4$, $1=3,2=2,3=1$ e $4=0$. As demais questões são negativas e devem ser somadas diretamente. O total da escala é a soma das pontuações destas 14 questões e os escores podem variar de zero a 56 .

Os idosos foram caracterizados em relação a outras variáveis baseadas nos estudos de Cohen et al ${ }^{1}(1983)$ e nas indicações de Spirduso, ${ }^{16}$ (2005) sobre fatores que influenciam no estresse do idoso. As questões incluem a auto-avaliação do estado de saúde, percepção da situação econômica, da memória, satisfação com a vida e acontecimento de eventos negativos. Estas questões foram utilizadas para comparar as médias do estresse percebido em função das variáveis apontadas na literatura, permitindo a visualização do uso da escala em pesquisas na área.

\section{Tradução e tradução reversa}

A tradução da escala de estresse percebido do inglês para o português foi realizada com base no protocolo proposto por Guillemin et $\mathrm{al}^{7}$ (1993): tradução inicial, tradução reversa e revisão por comitê de especialistas.

A tradução inicial do inglês para o português foi feita por dois tradutores independentes e qualificados que estavam cientes dos objetivos da tradução. A tradução reversa para o inglês (back-translation) foi realizada por outro tradutor independente bilíngüe, visando comparar a tradução para o inglês com o instrumento original. A etapa de revisão pelo comitê visou produzir uma versão final da escala, comparando os resultados entre si. O comitê foi formado por equipe multidisciplinar, composta por quatro profissionais da área da saúde pesquisadores desta linha de estudo. Durante o processo de tradução, foram observados os seguintes aspectos:

Equivalência semântica: avaliação da equivalência gramatical e de vocabulário. As palavras que não possuíam uma tradução literal com significado semelhante foram traduzidas para os termos em português que apresentavam equivalência de significado.

Equivalência idiomática: tradução de certas expressões idiomáticas que não pode ser feita de forma literal, devendo equivaler no seu sentido. As expressões foram traduzidas pelo seu sentido na língua inglesa, a expressão to be on top of things, por exemplo, foi traduzida para "estar com as coisas sob controle".

Equivalência experimental ou cultural: coerência entre os termos utilizados e as experiências vividas pela população à qual se destina, dentro do seu contexto cultural. A equivalência experimental ou cultural foi verificada por meio de entrevista com idosos, analisando qualitativamente as respostas. Esta fase da tradução foi realizada na segunda etapa desta pesquisa, por meio da análise da compreensão e adequação dos itens para o contexto dos idosos. 
Equivalência conceitual: muitos itens utilizados nos instrumentos podem equivaler-se semanticamente, sem, contudo, apresentar equivalência de conceito. Os termos podem representar conceitos diferentes em contextos específicos. A equivalência conceitual foi revisada na etapa em que a tradução foi revisada pelo comitê.

A tradução foi avaliada quanto à clareza para os idosos, analisando a compreensão com o grupo focal, aplicada pelos pesquisadores.

\section{Avaliação da tradução e validação inicial}

Participaram voluntariamente 76 idosos (70 mulheres e seis homens), com idade média de 70,04 anos ( $\mathrm{DP}=6,34$; mín: 60; máx: 84 ), pertencentes ao grupo de estudos da terceira idade.

A coleta de dados ocorreu durante três dias seguidos, em um ginásio de esportes de Florianópolis, Santa Catarina, das duas às quatro horas da tarde de outubro de 2005. Os entrevistadores se posicionaram no ginásio de forma a manter uma distância suficiente para não haver interferência. Os idosos foram entrevistados na ordem de chegada. As entrevistas foram realizadas individualmente, com duração média de $13,8(\mathrm{DP}=3,12)$ minutos, incluindo o questionário e a PSS. Antes do início da entrevista, foram explicados os procedimentos da pesquisa, sendo lido e assinado o Termo de Consentimento Livre e Esclarecido. Foi solicitado aos participantes que se manifestassem caso a questão não estivesse clara. As entrevistadoras apresentaram aos idosos uma folha impressa com as opções de resposta da escala, para que cada participante, ao ouvir a questão, pudesse visualizar as possibilidades de resposta. Caso o idoso não entendesse a questão, era lida novamente.

Os dados foram inseridos no pacote estatístico SPSS 11.0 para análises. A confiabilidade da escala foi avaliada pela consistência interna, verificada por meio do coeficiente alfa de Cronbach.

A validade de construto foi avaliada por meio de análise fatorial exploratória, que identifica os componentes comuns em um grande número de variáveis. Essa avaliação permite determinar o quanto a escala está relacionada aos conceitos teóricos que a fundamentam. A análise fatorial foi realizada de acordo com as etapas propostas por Dancey \& Reidy ${ }^{3}$ (2004).

Primeiramente, utilizou-se o método de componentes principais para extração dos fatores, considerando apenas aqueles que apresentaram eigenvalues superiores a 1. Os eigenvalues mostram a proporção de variância atribuída para cada valor. A soma dos eigenvalues é o número de variáveis na análise. Selecionados os fatores, foi gerada uma matriz correlacional, onde foram observadas as relações entre os itens e os fatores, por meio das cargas fatoriais.
Para a interpretação da matriz foi aplicado o método de rotação ortogonal varimax, que maximiza as correlações altas e minimiza as baixas, facilitando a análise. ${ }^{3}$

Os autores do instrumento original verificaram que as questões $4,5,12$ e 13 apresentaram cargas fatoriais baixas $(<0,48)$, optando por excluir estes itens para a elaboração da PSS com dez questões. ${ }^{2}$ Com base nestes resultados, optou-se por analisar a consistência interna (coeficiente alfa de Cronbach) e as cargas fatoriais da escala com 14 e com 10 questões.

Para verificar os resultados da aplicação da escala em relação a algumas variáveis apontadas na literatura, como: saúde, percepção da situação econômica, problemas de memória, entre outras, comparou-se as médias de estresse percebido resultante da escala em função das auto-avaliações dessas variáveis. Considerando que os dados apresentaram distribuição normal, as respostas que tinham mais de duas opções foram comparadas por meio de ANOVA e post-hoc de Scheffé.

As médias do índice de estresse percebido (ISP), em relação às perguntas que tinham apenas duas opções de resposta, foram comparadas por meio de teste $t$ para amostras independentes. O intervalo de confiança adotado foi de $95 \%$. Estes procedimentos foram feitos com base na validação realizada pelos autores da esca$1 a^{1,2}$ ao validar a PSS para a população americana. As qualidades psicométricas da escala com 14 questões foram comparadas com as da versão de dez questões, buscando verificar se a versão reduzida consegue medir o mesmo construto.

A pesquisa foi conduzida dentro dos padrões exigidos pela Declaração de Helsinki, e aprovada pelo Comitê de Ética em Pesquisa em Seres Humanos da Universidade do Estado de Santa Catarina, de acordo com a Resolução CNS 196/96.

\section{RESULTADOS}

O grupo focal de idosos sugeriu pequenas modificações na reformulação das questões 5 e 13. A questão 5 continha o termo "eficazmente", que foi substituído pelo advérbio "bem" (estar lidando bem com as mudanças). Na questão 13, o termo "gerenciar bem o tempo" foi substituído por "controlar a maneira como gasta o seu tempo". Após as modificações as questões foram consideradas claras e compreensíveis.

Contudo, o estudo de validação da PSS proporcionou o reconhecimento da necessidade de obedecer alguns cuidados durante a aplicação da escala. Por exemplo, a frase inicial, "Neste último mês, com que freqüência você...", deve ser dita antes de iniciar as perguntas e o entrevistador deve se certificar que o idoso entendeu que as perguntas se referiam à como ele vinha se sentindo no último mês. 
As opções de respostas da escala (nunca, quase nunca, às vezes, quase sempre e sempre) devem ser repetidas no final de todas as perguntas, caso a escala seja aplicada em forma de entrevista. Recomenda-se mostrar um papel impresso com estas opções em tamanho grande.

Após receber a resposta do idoso (no caso de entrevista), é aconselhado que o entrevistador leia a questão afirmativamente com a opção escolhida pelo entrevistado.

No caso de utilização da PSS como questionário autoaplicável, é necessário que o respondente leia atentamente as instruções antes de responder à escala.

Pela análise fatorial exploratória pelo Método dos Componentes Principais, verificou-se que dois fatores responderam por $48,1 \%$ da variância total da escala, sendo o fator 1 responsável por $34,6 \%$ da variação total (eigenvalue $=4,85$ ). $\mathrm{O}$ fator 2 foi responsável por $12,9 \%$ da variação total (eigenvalue $=1,89$ ). Na Tabela 1 são apresentadas cargas fatoriais de cada questão nos dois fatores extraídos da PSS.

Analisando a Tabela 1, observa-se que as questões diretamente relacionadas ao estresse percebido carregaram mais no fator 1 , enquanto que aquelas com conotação positiva $(4,5,6,7,9,10 \mathrm{e} 13)$, apresentaram as maiores cargas no fator 2 . No entanto, algumas destas questões carregaram também no fator 1 , mas de maneira menos significativa $(<0,48)$. A questão 12 não apresentou carga fatorial adequada em nenhum dos dois fatores, no entanto, a sua carga no fator 1 foi muito próxima a 0,48 , considerada aceitável, segundo os autores da escala.

Ao retirar as questões 4, 5, 12 e 13 para obter os valores da PSS 10, verificou-se aumento na consistência interna $(\alpha=0,83)$. De acordo com a análise fatorial, verificou-se que apenas um fator respondeu por $42,5 \%$ da variância total. Observou-se que todas as questões carregaram positivamente no fator 1, estando todas estas acima de 0,48 . Embora as questões positivas continuassem carregando no fator 2. A principal diferença entre a PSS 14 e a PSS 10 em relação às cargas fatoriais foi que todas as questões passaram a carregar significativamente no fator 1 .

A caracterização geral dos idosos é apresentada na Tabela 2, quanto às variáveis: sexo, escolaridade, estado civil, local de residência, bem como as auto-avaliações quanto à condição de moradia, saúde, esquecimentos, situação econômica atual comparada à anterior, renda mensal em relação às necessidades básicas (subjetivas), sentimento de estar feliz, estar mais irritado que o normal e acontecimento de evento emocional negativo importante.

Em relação ao resultado da PSS com 14 questões (PSS 14), a média geral apresentada pelos idosos foi de 21,37 $(\mathrm{DP}=7,699)$, variando de 7 a 41 pontos. Para a escala de estresse percebido com dez questões (PSS 10), a média geral foi de $15,13(\mathrm{DP}=6,459)$, variando de 5 a 33 .

Tabela 1. Cargas fatoriais para cada questão nos dois fatores extraídos da Escala de Estresse Percebido (PSS).

\begin{tabular}{|c|c|c|c|c|}
\hline \multicolumn{3}{|c|}{ Questão } & \multicolumn{2}{|c|}{ Carga fatorial } \\
\hline $\mathrm{N}^{\circ}$ & Neste último mês, com que freqüência... & $\mathrm{At}^{*}$ & Fator I & Fator II \\
\hline 2 & Você tem se sentido incapaz de controlar as coisas importantes em sua vida? & - & 0,736 & 0,117 \\
\hline 14 & $\begin{array}{l}\text { Você tem sentido que as dificuldades se acumulam a ponto de você acreditar que não } \\
\text { pode superá-las? }\end{array}$ & - & 0,716 & 0,356 \\
\hline 1 & Você tem ficado triste por causa de algo que aconteceu inesperadamente? & - & 0,704 & 0,127 \\
\hline 3 & Você tem se sentido nervoso e "estressado"? & - & 0,683 & $-0,021$ \\
\hline 11 & Você tem ficado irritado porque as coisas que acontecem estão fora do seu controle? & - & 0,672 & 0,153 \\
\hline 8 & Você tem achado que não conseguiria lidar com todas as coisas que você tem que fazer? & - & 0,491 & 0,418 \\
\hline 12 & Você tem se encontrado pensando sobre as coisas que deve fazer? & - & 0,470 & $-0,157$ \\
\hline 9 & Você tem conseguido controlar as irritações em sua vida? & + & 0,048 & 0,791 \\
\hline 6 & Você tem se sentido confiante na sua habilidade de resolver problemas pessoais? & + & 0,008 & 0,763 \\
\hline 13 & Você tem conseguido controlar a maneira como gasta seu tempo? & + & $-0,039$ & 0,624 \\
\hline 5 & $\begin{array}{l}\text { Você tem sentido que está lidando bem as mudanças importantes que estão ocorrendo em } \\
\text { sua vida? }\end{array}$ & + & 0,067 & 0,622 \\
\hline 4 & Você tem tratado com sucesso dos problemas difíceis da vida? & + & 0,300 & $\mathbf{0 , 5 8 7}$ \\
\hline 10 & Você tem sentido que as coisas estão sob o seu controle? & + & 0,392 & 0,522 \\
\hline \multirow[t]{2}{*}{7} & Você tem sentido que as coisas estão acontecendo de acordo com a sua vontade? & + & 0,386 & 0,492 \\
\hline & Coeficiente Alfa de Cronbach & & \multicolumn{2}{|c|}{0,82} \\
\hline
\end{tabular}

*At: significa atitude, sinais de negativo indicam soma normal e sinais de positivo indicam soma inversa.

Nota: Os valores em negrito destacam a carga fatorial mais elevada de cada questão. 
A comparação dos resultados da PSS com dez e com 14 questões em relação às variáveis está apresentada na Tabela 2. Verificou-se que as mulheres apresentam maior índice de estresse percebido do que os homens. Entretanto, a diferença foi significativa apenas para a comparação das médias da PSS $10(\mathrm{t}=-2,143$; $\mathrm{p}=0,035)$.
Em relação à escolaridade, verificou-se que quanto maior a escolaridade, menor o nível de estresse. No entanto, não houve diferenças estatísticas entre as médias dos grupos, tanto na PSS $14(\mathrm{~F}=2,163 ; \mathrm{p}=0,082)$ quanto na PSS $10(\mathrm{~F}=2,092 ; \mathrm{p}=0,091)$. Em relação ao estado civil, não foram verificadas diferenças entre os grupos.

Tabela 2. Médias de estresse percebido em função das variáveis do questionário de estresse do idoso. Florianópolis, SC, 2005.

\begin{tabular}{|c|c|c|c|c|c|c|}
\hline \multirow[b]{2}{*}{ Variável } & \multirow[b]{2}{*}{ Categoria } & \multirow[b]{2}{*}{$\mathrm{N}$} & \multicolumn{2}{|c|}{ PSS 14} & \multicolumn{2}{|c|}{ PSS 10} \\
\hline & & & Média & Desvio-padrão & Média & Desvio-padrão \\
\hline \multirow[t]{4}{*}{ Gênero* } & Feminino & 70 & 21,83 & 7,701 & $15,59 *$ & 6,446 \\
\hline & Masculino & 6 & 16,00 & 5,76 & 9,83 & 3,971 \\
\hline & Nenhuma & 8 & 28,25 & 6,274 & 20,50 & 5,099 \\
\hline & Primário & 30 & 21,23 & 7,295 & 15,13 & 6,185 \\
\hline \multirow[t]{4}{*}{ Escolaridade } & $1^{\circ} \mathrm{grau}$ & 9 & 21,00 & 5,292 & 15,11 & 4,045 \\
\hline & $2^{\circ}$ grau & 15 & 20,60 & 8,147 & 14,73 & 7,259 \\
\hline & Curso superior & 14 & 18,79 & 6,066 & 12,50 & 5,694 \\
\hline & Casado & 36 & 21,97 & 7,424 & 15,42 & 6,398 \\
\hline \multirow[t]{3}{*}{ Estado civil } & Viúvo & 31 & 21,45 & 8,842 & 15,39 & 7,191 \\
\hline & Divorciado & 3 & 18,00 & 3,606 & 11,67 & 4,163 \\
\hline & Solteiro & 6 & 19,00 & 3,578 & 13,83 & 3,430 \\
\hline \multirow[t]{2}{*}{ Local de residência } & Casa & 33 & 22,73 & 7,234 & 16,42 & 5,922 \\
\hline & Apartamento & 42 & 19,93 & 7,617 & 13,81 & 6,463 \\
\hline \multirow[t]{3}{*}{ Condições de moradia } & Ótimo & 47 & 20,00 & 7,729 & 14,19 & 6,566 \\
\hline & Bom & 24 & 23,21 & 7,301 & 16,58 & 6,107 \\
\hline & Regular & 2 & 28,50 & 6,364 & 20,50 & 4,950 \\
\hline \multirow[t]{2}{*}{ Problemas de saúde* } & Sim & 54 & $22,59 *$ & 7,818 & 16,00 & 6,636 \\
\hline & Não & 22 & 18,36 & 6,644 & 13,00 & 5,581 \\
\hline \multirow[t]{4}{*}{ Auto-avaliação da saúde* } & Ótima & 12 & 16,17 & 8,548 & 11,50 & 7,775 \\
\hline & Boa & 37 & 21,41 & 6,681 & 15,16 & 5,776 \\
\hline & Regular & 22 & $23,55^{*}$ & 7,866 & 16,59 & 6,299 \\
\hline & Ruim & 5 & 24,00 & 8,246 & 17,20 & 7,120 \\
\hline \multirow[t]{4}{*}{ Situação econômica } & Melhor & 37 & 22,46 & 7,705 & 16,22 & 6,365 \\
\hline & A mesma & 20 & 18,60 & 6,924 & 12,55 & 5,596 \\
\hline & Pior & 19 & 22,16 & 8,126 & 15,74 & 7,038 \\
\hline & Dá e sobra & 22 & 19,81 & 8,387 & 14,32 & 6,910 \\
\hline \multirow[t]{3}{*}{ Necessidades básicas } & Dá na conta certa & 35 & 20,77 & 6,517 & 14,57 & 5,648 \\
\hline & Sempre falta um pouco & 15 & 23,27 & 9,020 & 16,53 & 7,836 \\
\hline & Sempre falta muito & 4 & 28,00 & 5,477 & 19,25 & 4,425 \\
\hline \multirow[t]{3}{*}{ Satisfação com a vida* } & Satisfeito & 62 & 20,19 & 7,494 & 14,21 & 6,302 \\
\hline & Mais ou menos & 14 & $26,57^{*}$ & 6,536 & 19,21 & 5,686 \\
\hline & Insatisfeito & 22 & 20,00 & 7,838 & 14,00 & 6,789 \\
\hline \multirow[t]{2}{*}{ Esquecimentos* } & Às vezes & 32 & $19,97^{*}$ & 7,351 & 14,44 & 5,967 \\
\hline & Quase sempre & 22 & 24,77 & 7,309 & 17,27 & 6,591 \\
\hline \multirow[t]{3}{*}{ Sentir-se feliz* } & Sim & 56 & 19,71 & 7,039 & 13,86 & 6,019 \\
\hline & Mais ou menos & 18 & $25,39 *$ & 7,935 & $18,06^{*}$ & 6,485 \\
\hline & Não & 2 & 31,50 & 0,707 & 24,50 & 0,707 \\
\hline \multirow[t]{2}{*}{ Mais irritado que o normal* } & Sim & 29 & $26,41^{*}$ & 7,529 & $19,52^{*}$ & 6,512 \\
\hline & Não & 47 & 18,26 & 6,016 & 12,43 & 4,754 \\
\hline \multirow[t]{2}{*}{ Evento emocional* } & Sim & 45 & $24,07^{*}$ & 7,620 & $17,44^{*}$ & 6,419 \\
\hline & Não & 31 & 17,45 & 6,021 & 11,77 & 4,917 \\
\hline
\end{tabular}

* diferença estatisticamente significativa $(p<0,05)$ 
Os idosos que residem em casas não apresentaram escores diferentes daqueles que moram em apartamentos. A diferença da média do estresse percebido em relação à avaliação dos idosos sobre o local onde moram também não foi estatisticamente significativa entre as versões (PSS 14: $F=1,868 ; p=0,143$ ) (PSS 10: $\mathrm{F}=1,216 ; \mathrm{p}=0,310$ ), embora se possa verificar que os idosos que percebiam o local com ótimo apresentaram uma média de estresse percebido inferior à apresentada pelos demais.

Os idosos que indicaram ter problemas de saúde obtiveram média de estresse percebido superior aos que não indicaram doenças. Esta diferença foi significativa na PSS $14(\mathrm{t}=2,228 ; \mathrm{p}=0,029)$, mas não na PSS 10 $(\mathrm{t}=1,867 ; \mathrm{p}=0,066)$.

Em relação à auto-avaliação da saúde, os idosos que percebem a sua saúde como regular apresentaram maior estresse do que os que a consideraram boa ou ótima. Estas diferenças foram estatisticamente significativas na PSS $14(\mathrm{~F}=2,872 ; \mathrm{p}=0,042)$, mas não na PSS 10 $(\mathrm{F}=1,873 ; \mathrm{p}=0,142)$.

O estresse percebido não foi estatisticamente diferente em função da situação econômica atual. Os idosos que indicaram estar melhor financeiramente em relação à quando tinham 50 anos de idade apresentaram estresse percebido similar ao apresentado pelos que relataram piora na situação econômica. No entanto, a avaliação da renda mensal para suprir as necessidades básicas diferenciou a média de estresse percebido dos idosos, embora esta diferença não tenha sido estatisticamente significativa.

Os idosos que referiram estar satisfeitos com as suas vidas mostraram estresse percebido significativamente menor do que os que relataram estar "mais ou menos" satisfeitos ou insatisfeitos, tanto na PSS $14(\mathrm{~F}=4,260$; $\mathrm{p}=0,018)$ quanto na PSS $10(\mathrm{~F}=3,719 ; \mathrm{p}=0,029)$.

Os idosos que relataram estar felizes também mostraram índice de estresse significativamente inferior ao daqueles "mais ou menos" felizes, ou infelizes, tanto na PSS $14(\mathrm{~F}=6,245 ; \mathrm{p}=0,003)$, quanto na PSS 10 $(\mathrm{F}=5,666 ; \mathrm{p}=0,005)$.

A resposta "estar mais irritado que o normal" parece influenciar na média de estresse percebido na PSS 14 $(\mathrm{t}=5,212 ; \mathrm{p}<0,001)$ e na PSS $10(\mathrm{t}=5,666 ; \mathrm{p}=0,005)$. Os idosos que relataram a ocorrência de um "evento emocional que ainda o deixa triste" obtiveram uma média de estresse percebido significativamente superior àqueles que não indicaram a presença de tais eventos, tanto na PSS $14(\mathrm{t}=4,040 ; \mathrm{p}<0,001)$ quanto na PSS 10 $(\mathrm{t}=4,148 ; \mathrm{p}<0,001)$.

Em relação aos esquecimentos, foi verificado que os idosos que indicaram "quase sempre" esquecer coisas apresentaram estresse percebido significativamente superior ao daqueles que relataram esquecer raramente ou às vezes. No entanto, estas diferenças foram verificadas apenas na PSS $14(\mathrm{~F}=3,205 ; \mathrm{p}=0,041)$ e não na PSS 10 $(\mathrm{F}=1,767 ; \mathrm{p}=0,178)$.

\section{DISCUSSÃO}

No presente estudo, o número de participantes do sexo feminino e masculino foi desigual. Os resultados referentes às diferenças dos índices de estresse percebido entre os sexos podem ter sido suscetíveis a erros tipo I.

Devido à distribuição desigual nas opções de auto-avaliação, em perguntas com cinco opções de respostas, foi difícil verificar a significância estatística de algumas comparações. Entre elas, as que relacionavam índice de estresse percebido e situação socioeconômica, escolaridade, auto-avaliação da saúde, entre outras.

Em geral, a tradução da PSS (Anexo) apresentou resultados satisfatórios, após ter sido modificada e testada empiricamente em um grupo de idosos. Após essas modificações, a escala mostrou-se válida quanto à clareza. Também apresentou boa confiabilidade e validade de construto, evidenciado pelo coeficiente encontrado e pelas cargas fatoriais.

A PSS vem sendo validada em diversos países, como nos Estados Unidos, ${ }^{1,2}$ Japão ${ }^{11}$ e Espanha. ${ }^{14}$ Foi observada a concordância de resultados da presente pesquisa com tais estudos, especialmente aos referentes à validade de construto.

Na PSS 14, verificou-se que dois fatores responderam pela maior parte da variância com eigenvalues acima de 1 . A existência destes dois fatores e a variação atribuída a cada um foi semelhante à encontrada nos outros estudos de validação da PSS. ${ }^{2,8,11}$

As cargas fatoriais encontradas no presente estudo foram semelhantes às encontradas por Mimura \& Griffiths ${ }^{11}(2004)$, especialmente para a versão original em inglês. Esses autores ${ }^{11}$ destacam que o fator 1 pode ser descrito como "percepção negativa", enquanto o fator 2 deve ser interpretado como "percepção positiva". Hewitt et al ${ }^{8}(1992)$ ao verificar a presença destes dois fatores, denominou o fator 1 como "distress percebido" e o fator 2 como "coping percebido". Estas denominações são confirmadas ao observar que embora as questões sejam colocadas no banco de dados conforme são somadas (as positivas são somadas invertidas), dois fatores são gerados, de forma que as questões negativas carregam no fator 1, enquanto as questões positivas carregam no com mais força no fator 2 .

Uma das questões retiradas PSS 14 para formar a PSS 10 é a de número 12. Esta foi a que apresentou a menor 
carga fatorial, concordando com o achado de Cohen \& Williamsom $^{2}$ (1988), que verificou baixa carga fatorial para esta questão. Mimura \& Griffiths ${ }^{11}$ (2004) também obtiveram cargas fatoriais baixas nesta questão.

Ao realizar a análise fatorial exploratória com a PSS 10, foi extraído apenas um fator e todas as questões apresentaram cargas fatoriais acima de 0,48. Estes resultados indicam que a PSS 10 apresenta uma melhor adequação fatorial, concordando com os estudos anteriores. ${ }^{2,11}$

A comparação das cargas fatoriais do presente estudo com as encontradas em pesquisas em outras culturas indica a validade da tradução e a possibilidade de utilização da PSS para estudos multiculturais.

Os resultados da aplicação da PSS encontrados na presente pesquisa concordam com os de outros estudos de validação, ${ }^{1}$ embora maiores que as médias de estresse verificadas por Cohen et $\mathrm{al}^{1}$ (1983). Por exemplo, foi verificado que o estresse percebido médio de pessoas com mais de 65 anos era de $18,5(\mathrm{DP}=7,8)$, enquanto que no presente estudo, a média geral foi de 22,02 $(\mathrm{DP}=8,00)$.

A diferença no nível de estresse entre os participantes do presente estudo e os idosos americanos ${ }^{1}$ pode ser explicado por algumas razões, por exemplo, a pesquisa americana foi realizada há 24 anos. Os problemas enfrentados pelos idosos brasileiros atualmente são, provavelmente, diferentes daqueles vivenciados pelos americanos em 1983. Além disso, o número de participantes brasileiros foi inferior ao estudado por Cohen et al. ${ }^{1}$ Para saber como está o estresse percebido dos idosos brasileiros em relação ao verificado em outros países, serão necessários estudos populacionais.

Os resultados da PSS 14 e PSS 10 foram similares. No entanto, ao comparar as médias de estresse percebido por meio de testes inferenciais, o índice obtido com a
PSS 14 possibilitou diferenciar mais os grupos. Esta diferença pode indicar que embora a PSS 14 apresente consistência interna levemente inferior $(\mathrm{r}=0,82)$ à PSS $10(\mathrm{r}=0,83)$ e adequação fatorial distinta, ela parece ser mais eficaz para detectar as diferenças entre os grupos.

Em relação aos resultados da escala, é comum a prática de enquadrar os escores em categorias, como: baixo, médio, alto. No entanto, os autores da $\mathrm{PSS}^{1}$ não recomendam esta prática, pois ao se agrupar diferentes escores em uma mesma categoria, perde-se precisão nas análises estatísticas.

Alguns pesquisadores categorizam as variáveis justificando que a maioria das decisões clínicas é dicotômica e, portanto, os resultados dos testes e escalas devem ser enquadrados em diferentes classificações. Dancey $\& \operatorname{Reidy}^{3}$ (2004) afirmam que classificar ou agrupar escores de variáveis contínuas é um erro que leva a uma perda de sensibilidade na análise dos resultados, tanto na pesquisa quanto na prática clínica. Por esta razão, não são sugeridos pontos de corte, pois se entende o estresse percebido como uma variável contínua, que deve ser analisada como tal. No entanto, Streiner ${ }^{17}$ (2002) argumenta que não é correto analisar as decisões clínicas como dicotômicas, pois na prática, as avaliações estão baseadas em análise de um continuum. Ao analisar os escores de uma variável contínua ganha-se precisão, permitindo maior análise em um conjunto de fatores individuais, possibilitando avaliação mais precisa.

As características psicométricas da PSS 14 e da PSS 10 preencheram os critérios de consistência interna, validade de construto e apresentaram resultados semelhantes aos que deram origem à versão original da escala e às validações posteriores em diferentes culturas. Por estes motivos, conclui-se que a versão brasileira da PSS é válida para mensurar o estresse percebido de idosos brasileiros, para uso em estudos epidemiológicos e multiculturais. 


\section{REFERÊNCIAS}

1. Cohen S, Karmack T, Mermelsteinm R. A global measure of perceived stress. I Health Soc Behav. 1983;24(4):385-96.

2. Cohen S, Williamsom GM. Perceived Stress in a Probability Sample of United States. In: Spacapan S, Oskamp S, editores. The Social Psychology of Health: Claremont Symposium on applied social psychology. Newbury Park, CA: Sage; 1988.

3. Dancey CP, Reidy J. Statistics without maths for Psychology: using SPSS for Windows. 3.ed. London: Prentice Hall; 2005.

4. Goldman N, Glei DA, Seplaki C, Liu IW, Weinstein $M$. Perceived stress and physiological dysregulation in older adults. Stress. 2005;8(2):95-105.

5. Grace SL, Krepostman S, Brooks D, Arthur H, Scholey $\mathrm{P}$, Suskin N, et al. Illness perceptions among cardiac patients: relation to depressive symptomatology and sex. J Psychosom Res. 2005 59(3):153-60.

6. Greenberg JS. Administração do estresse. São Paulo: Manole; 2002.

7. Guillemin F, Bombardier C, Beaton D. Cross-cultural adaptation of health-related quality of life measures: literature review and proposed guidelines. J Clin Epidemiol. 1993;46(12):1417-32.

8. Hewitt PL, Flett GL, Mosher SW. The perceived stress scale: factor structure and relation to depression symptoms in a psychiatric sample. J Psychopathol Behav Assess. 1992;14(2):247-57.

9. Lazarus RS, Folkman L. Stress, coping and adaptation. New York: Springer; 1984.

10. Maia LC, Durante AMG, Ramos LR. Prevalência de transtornos mentais em área urbana no norte de Minas Gerais, Brasil. Rev Saude Publica. 2004;38(5):650-6.

11. Mimura C, Griffiths P. A Japanese version of the perceived stress scale: translation and preliminary test. Int J Nurs Stud. 2004;41(4):379-85.
12. Porcu M, Scantamburlo VM, Albrecht NR, Silva SP, Vallim FL, Araújo CR, et al. Estudo comparativo sobre a prevalência de sintomas depressivos em idosos hospitalizados, institucionalizados e residentes na comunidade. Acta Scientiarum. 2002;24(3):713-7.

13. Reiche EM, Nunes SO, Morimoto HK. Stress, depression, the immune system, and cancer. Lancet Oncol. 2004 5(10):617-25

14. Remor E. Psychometric properties of a European Spanish Version of the Perceived Stress Scale (PSS). Span J Psychol. 2006;9(1):86-93.

15. Rush AJ, Zimmerman M, Wisniewski SR, Fava M, Hollon SD, Warden D, et al. Comorbid psychiatric disorders in depressed outpatients: demographic and clinical features. J Affect Disord. 2005;87(1):43-55.

16. Spirduso WW. Dimensões físicas do envelhecimento. Trad. de P Bernardi. Barueri: Manole, 2005.

17. Streiner DL. Breaking up is hard to do: The heartbreak of dichotomizing continuous data. Can J Psychiatry. 2002;47(3):262-6

18. Strik JJMH, Denollet J, Lousberg R, Honig A. Comparing symptoms of depression and anxiety as predictors of cardiac events and increased health care consumption after myocardial infarction. J Am Coll Cardiol. 2003;42(10):1801-7.

19. Van Winkle E. The toxic mind: the biology of mental illness and violence. Med Hypotheses. 2000;54(1):146-56.

20. Wang JJ, Snyder M, Kaas M. Stress, loneliness, and depression in Taiwanese rural community-dwelling elders. Int J Nurs Stud. 2001;38(3):339-47.

21. Xavier FMF, Ferraz MPT, Trenti CM, Argimon I, Bertollucci PH, Poyares D, et al. Transtorno de ansiedade generalizada em idosos com oitenta anos ou mais. Rev Saude Publica. 2001;35(3):294-302.

CDB Luft foi apoiada pela Coordenação de Aperfeiçoamento de Pessoal de Nível Superior (Capes - bolsa de mestrado). 


\section{ANEXO}

\section{ESCALA DE ESTRESSE PERCEBIDO}

\section{Itens e instruções para aplicação}

As questões nesta escala perguntam sobre seus sentimentos e pensamentos durante o último mês. Em cada caso, será pedido para você indicar o quão freqüentemente você tem se sentido de uma determinada maneira. Embora algumas das perguntas sejam similares, há diferenças entre elas e você deve analisar cada uma como uma pergunta separada. A melhor abordagem é responder a cada pergunta razoavelmente rápido. Isto é, não tente contar o número de vezes que você se sentiu de uma maneira particular, mas indique a alternativa que lhe pareça como uma estimativa razoável. Para cada pergunta, escolha as seguintes alternativas:

$0=$ nunca

$1=$ quase nunca

$2=$ às vezes

$3=$ quase sempre

$4=$ sempre

\begin{tabular}{|c|c|c|c|c|c|c|}
\hline \multicolumn{7}{|c|}{ Neste último mês, com que freqüencia... } \\
\hline 1 & Você tem ficado triste por causa de algo que aconteceu inesperadamente? & 0 & 1 & 2 & 3 & 4 \\
\hline 2 & Você tem se sentido incapaz de controlar as coisas importantes em sua vida? & 0 & 1 & 2 & 3 & 4 \\
\hline 3 & Você tem se sentido nervoso e "estressado"? & 0 & 1 & 2 & 3 & 4 \\
\hline 4 & Você tem tratado com sucesso dos problemas difíceis da vida? & 0 & 1 & 2 & 3 & 4 \\
\hline 5 & $\begin{array}{l}\text { Você tem sentido que está lidando bem as mudanças importantes que estão ocorrendo em } \\
\text { sua vida? }\end{array}$ & 0 & 1 & 2 & 3 & 4 \\
\hline 6 & Você tem se sentido confiante na sua habilidade de resolver problemas pessoais? & 0 & 1 & 2 & 3 & 4 \\
\hline 7 & Você tem sentido que as coisas estão acontecendo de acordo com a sua vontade? & 0 & 1 & 2 & 3 & 4 \\
\hline 8 & Você tem achado que não conseguiria lidar com todas as coisas que você tem que fazer? & 0 & 1 & 2 & 3 & 4 \\
\hline 9 & Você tem conseguido controlar as irritações em sua vida? & 0 & 1 & 2 & 3 & 4 \\
\hline 10 & Você tem sentido que as coisas estão sob o seu controle? & 0 & 1 & 2 & 3 & 4 \\
\hline 11 & Você tem ficado irritado porque as coisas que acontecem estão fora do seu controle? & 0 & 1 & 2 & 3 & 4 \\
\hline 12 & Você tem se encontrado pensando sobre as coisas que deve fazer? & 0 & 1 & 2 & 3 & 4 \\
\hline 13 & Você tem conseguido controlar a maneira como gasta seu tempo? & 0 & 1 & 2 & 3 & 4 \\
\hline 14 & $\begin{array}{l}\text { Você tem sentido que as dificuldades se acumulam a ponto de você acreditar que não pode } \\
\text { superá-las? }\end{array}$ & 0 & 1 & 2 & 3 & 4 \\
\hline
\end{tabular}

